\title{
Acute oxalate nephropathy
}

\author{
WT Hinchliffe, S Fenwick and K MacDougall
}

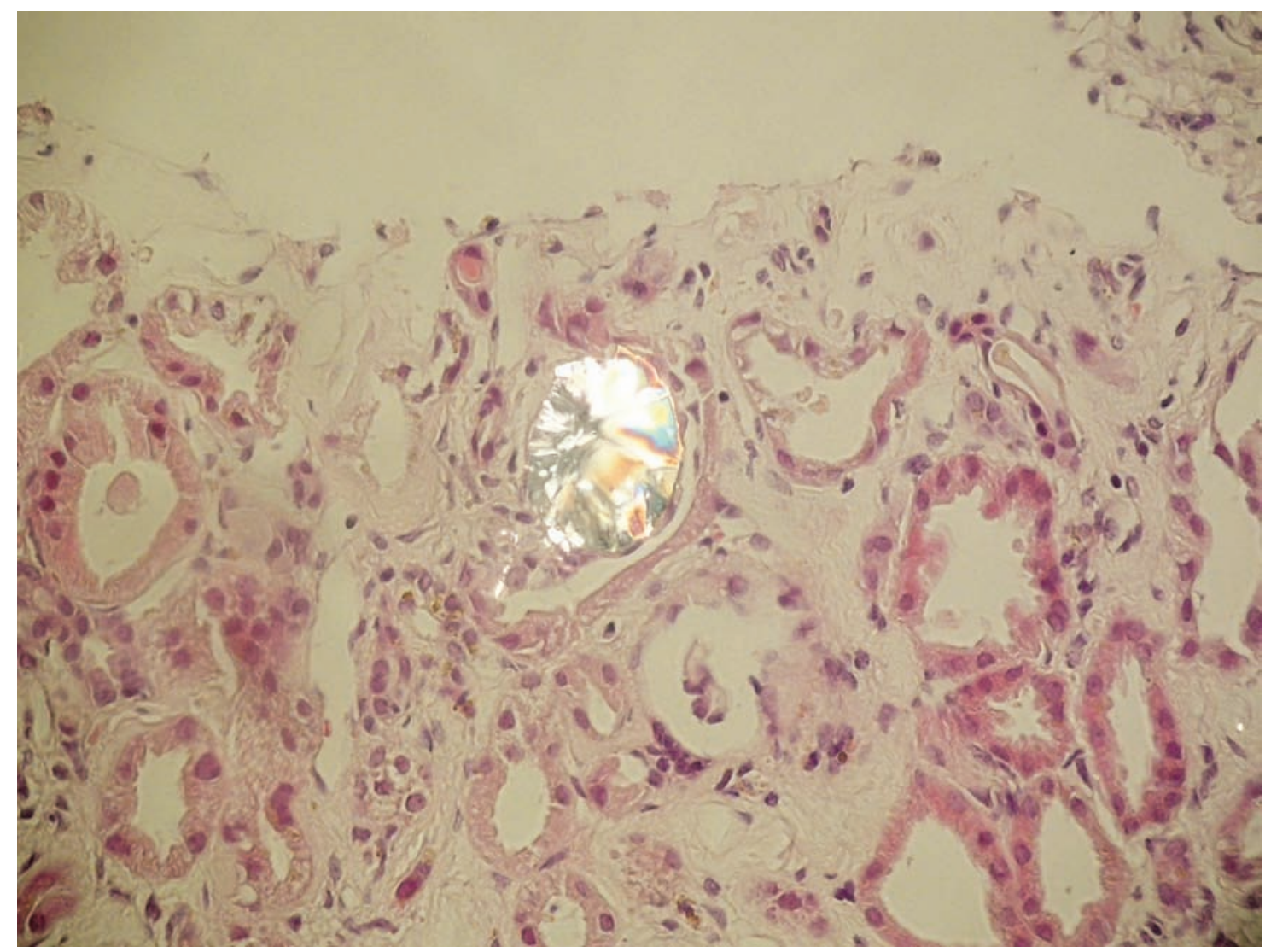

Fig 1. Renal biopsy of patient with acute kidney injury.

KEY WORDS: oxalate, renal failure, nephropathy, Orlistat, enteric hyperoxalurine

We were referred a patient at a stage of partial resolution of acute kidney injury (peak serum creatinine 590 umol/l). A renal biopsy was performed (Fig 1). Acute oxalate nephropathy was diagnosed and Orilstat $\left(\mathrm{Xenical}^{\circledR}\right)$ was considered causal. Orlistat has been available over the counter in the UK since 2009 and its usage will increase. We highlight this adverse reaction and advocate monitoring biochemistry.

Address for correspondence: Dr WT Hinchliffe,

Sunderland Royal Infirmary,

Kayll Road, Sunderland, Tyne \& Wear, SR4 7TP.

Email: Will22@doctors.org.uk

WT Hinchliffe, renal registrar; S Fenwick, consultant nephrologist;

K MacDougall, consultant histopathologist

City Hospitals Sunderland Foundation NHS Trust, UK 